\title{
Les mécanismes tensio-actifs à la surface des alvéoles pulmonaires
}

Le surfactant est un mélange complexe de phospholipides et d'apoprotéines qui permet de diminuer la tension d'interface eau/air au niveau des alvéoles pulmonaires et donc d'empêcher leur collapsus en expiration et d'améliorer l'ensemble de la mécanique respiratoire. L'immaturité pulmonaire des prématurés est associée à une carence en surfactant, mécanisme essentiel de la maladie des membranes hyalines. La mise au point de succédanés du surfactant, permise par la connaissance biochimique et physiologique du système, représente un important progrès pour traiter le syndrome de détresse respiratoire qu'entraîne cette maladie.

\section{Benoît-André Denizot Pierre-Cyril Tchoreloff Laurent-Michel Bonanno Jacques-Émile Proust Albert Lindenbaum Michel Dehan Francis Puisieux}

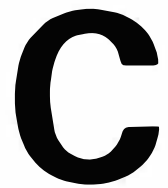
aractérisée cliniquement par une détresse respiratoire, la maladie des membranes hyalines (MMH) du nouveau-né touche, en France, environ 5000 enfants par an. Faible pour les enfants à terme, son incidence atteint $80 \%$ chez les prématurés de moins de 28 semaines [1]. Le développement de techniques de réanimation néonatales (oxygénothérapie, ventilation assistée) a permis d'améliorer le pronostic vital, autrefois gravissime. Cependant, les lacunes de cette réanimation, iatrogénicité (essentiellement dysplasie bronchopulmonaire) et inefficacité relative pour les grands prématurés, incite au développement de recherches dans le but de mieux traiter ces enfants. Chez les enfants souffrant de $\mathrm{MMH}$, l'immaturité fonctionnelle biochimique des poumons ne permet pas une synthèse suffisante de surfactant. Le surfactant pulmonaire est défini comme l'ensemble des substances tensioactives présentes physiologiquement à l'interface air - sous-phase alvéolaire. La thérapeutique substitutive à l'aide de surfactant exogène se propose de compenser le déficit en surfactant endogène par l'administration intrapulmonaire de substances exogènes, capables de jouer in vivo le rôle du surfactant naturel.

Les surfactants exogènes naturels (provenant de prélèvements biologiques, dont le chef de file est un extrait de poumons de bœuf modifié (Beractant ${ }^{\circledR}$ ou Survanta ${ }^{\circledR}$, laboratoires Abbott), sont efficaces cliniquement à court terme [2] du point de vue pronostic vital et fonctionnel (réduction des complications de la ventilation artificielle), mais posent des problèmes de disponibilité et de risques d'utilisation (immunogénicité [3], contamination par des microorganismes, reproductibilité des lots). Les surfactants semi-synthétiques, qui sont une recombinaison de lipides synthétiques et d'apoprotéines spécifiques [4], offrent une disponibilité acceptable, mais exposent pratiquement aux mêmes risques que les surfactants naturels.

Les surfactants artificiels se distinguent des autres classes par l'absence totale de composants d'origine natu- 


\section{RÉFÉRENCES}

1. Dehan M. Maladie des membranes hyalines du prématuré : aspects diagnostiques et perspectives thérapeutiques, ventilation mécanique exclue. Rev Prat 1989; 39 : 1844-8.

2. Horbar JD, Soll RF, Sutherland JM, et al. A multicenter randomized, placebocontrolled trial of surfactant therapy for respiratory distress syndrome. $N$ Engl J Med $1989 ; 320$ : 959-65.

3. Strayer DS, Merritt TA, LwebugaMukasa J, Hallman M. Surfactant - antisurfactant immune complexes in infants with respiratory distress syndrome. $\mathrm{Am} \mathrm{J}$ Pathol 1986 ; 122 : 353-62.

4. Hawgood S. Pulmonary surfactant apoproteins : a review of protein and genomic structure. Am J Physiol 1989; 257 : L13-22.

5. Waring A, Taeusch W, Bruni R, et al. Synthetic amphipathic sequences of surfactant protein- $B$ mimic several physicochemical and in vivo properties of native pulmonary surfactant proteins. Peptide Res 1989 2 : 308-13.

6. Von Neergard K. Neue Auffassungen über einen Grundbegriff der Atemmechanik: Die Retraktionskraft der Lunge, abhängig von der Oberflächenspannung in den Alveolen. $Z$ Ges Exp Med 1929 ; 66 : 373-94.

7. Guyton AC, Moffat DS, Adair TH Role of alveolar surface tension in transepithelial movement of fluid. In : Robertson B, Van Golde LMG, Batenburg JJ, eds. Pulmonary Surfactant, Amsterdam : Elsevier, $1984: 57-72$

8. Schürch S, Goerke J, Clements JA. Direct determination of surface tension in the lung. Proc Nall Acad Sci USA 1976; 73 4698-702.

9. Smith JC, Stamenovic D. Surface forces in lungs. I. Alveolar surface tension in the lung volume relationships. $J A p p l$ Physiol $1986 ; 60: 1341-50$.

10. Tchoreloff P, Denizot B, Proust JE Puisieux F. Induced structures obtained from surface film compression of natural lung surfactant. In : Herzog $\mathrm{H}$, ed. Basic Research on Lung Surfaclant, Progress in Respiration Research. Basel : Karger, 1990: 168-75. relle. Ils comprennent le plus souvent de la DPPC (dipalmitoylphosphatidylcholine). Avec les premiers produits testés en clinique, l'action respiratoire était partielle et s'épuisait en général en moins de 48 heures. L'Exosurf $^{\circledR}$ (un mélange de DPPC, d'hexadécanol et de Tyloxapol ${ }^{\circledR}$ produit par les laboratoires Wellcome), testé en clinique actuellement à grande échelle, semble notablement plus performant tant au niveau de la physiologie respiratoire (augmentation de la compliance ventilatoire et amélioration de l'hématose) que de l'amélioration du pronostic vital et fonctionnel $^{*}$, sans semble-t-il, atteindre l'efficacité des surfactants naturels. Les avantages de ces produits artificiels (risques infectieux faibles, risques immunogènes nuls, bonne disponibilité, coût modéré) incitent à continuer les recherches dans cette direction.

\section{Présentation du surfactant}

Avant d'étudier les mécanismes tensioactifs du surfactant, quelques notions sont à rappeler.

- US and Canadian Exosurf Pediatric Study Group. Effects of two rescue doses of Exosurf Pedialric in large infants with RDS. Poster at the " 60 years of surfactant research " congress, Rhine river, 11th-17th November 1989
La composition du surfactant est extrêmement complexe, comme le montre le Tableau I, sur lequel figure la composition moyenne actuellement admise. Le surfactant pulmonaire naturel présente trois particularités : (1) l'importance quantitative des phosphatidylcholines disaturées, essentiellement de la DPPC (dipalmitoylphosphatidylcholine) $(\approx 50 \% \mathrm{du}$ poids); (2) la quasi-absence des phospholipides habituellement trouvés dans les membranes cellulaires (phosphatidyléthanolamine, sphingomyéline, phosphatidylsérine) et la présence de phosphatidylglycérol PG ( $\approx 8 \% \mathrm{du}$ poids), phospholipide anionique très commun chez les bactéries mais spécifique, chez les mammifères, du surfactant pulmonaire ; (3) la présence d'apoprotéines spécifiques SP-A, -B et -C (SP : surfactant protein) [4].

La SP-A est une glycoprotéine hydrophile de $36 \mathrm{kDa}$ pour le monomère, comportănt une extrémité glycosylée et une portion collagénique ayant une structure native octadécamérique de $700 \mathrm{kDa}$ très proche de celles du facteur du complément du C1q ou de la CRP ( $C$ reactive protein). La SP-A possède la particularité de s'agréger en présence de $\mathrm{Ca}++$ et est partiellement liée à la portion lipidique du surfactant, l'insertion dans la couche lipidique se faisant par l'extrémité non glycosylée.

\section{Tableau}

COMPOSITION CHIMIQUE DU SURFACTANT PULMONAIRE (en $\%$ de poids)

Phospholipides
Phosphatidylcholines saturées
Phosphatidylcholines insaturées
Phosphatidylglycérol
Phosphatidylinositol
Phosphatidyléthanolamine
Sphingomyéline
Autres
Lipides neutres et cholestérol
Protéines
Protéines "sériques ": albumine...
SP-A ( $\approx 35 \mathrm{kDa}$, glycosylée)
SP-B $(8-18 \mathrm{kDa})$, SP-C ( $\approx 5 \mathrm{kDa})$

[Jobe et Ikegami, Am Rev Resp Dis 1987; 136: 1256-75.]
85

50
18
8
2
4
1
2


8
1
1

$m / s$ n 1 , vol 7 janvier 91 


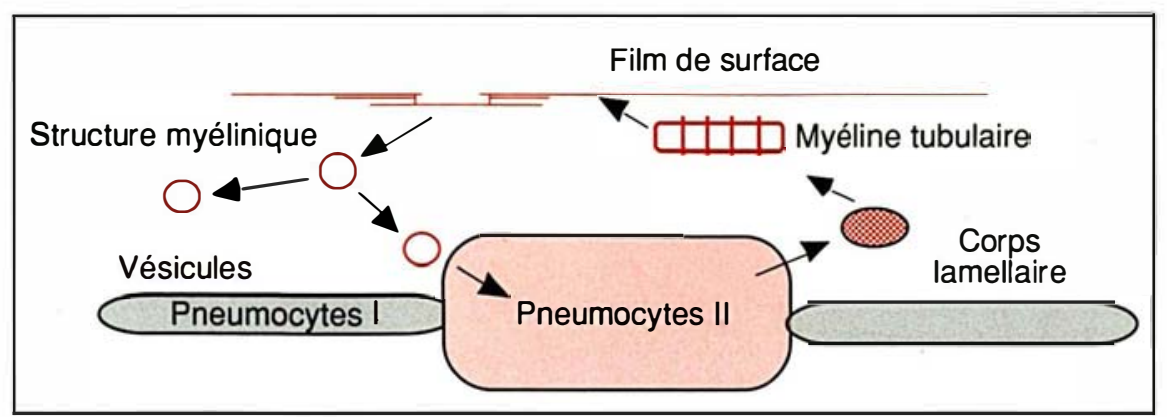

Figure 1. Schéma fonctionnel de la morphologie de la sous-phase alvéolaire. Les particules de surfactant, synthétisées par les pneumocytes II, sont excrétées sous la forme de corps lamellaires, puis sont transformées en myéline tubulaire, qui est un précurseur direct de la couche tensio-active et des structures myéliniques. De nombreuses vésicules, structures d'élimination des phospholipides, sont recyclées par les pneumocytes II.

La SP-B est une protéine lipophile, extraite avec les lipides lors d'une purification en milieu organique. Son poids moléculaire est de 8 à $18 \times$ $10^{3}$ par électrophorèse en milieu dénaturant (SDS-PAGE) et de 7,8 $\times$ $10^{3}$ par analyse des acides aminés. La conformation de cette protéine non glycosylée, uniquement membranaire, est celle d'un amphiphile.

La SP-C est également lipophile, d'un poids moléculaire d'environ 5 $\times 10^{3}$ en SDS-PAGE et de $3,7 \times$ $10^{3}$ par analyse des acides aminés. Aussi bien pour la SP-B que pour la SP-C, la charge cationique portée par ces protéines semble importante pour la conformation spatiale [5].

Les particules de surfactant, excrétées par les pneumocytes II, se trouvent sous diverses formes dans la sousphase alvéolaire (figure 1): corps lamellaires (particules arrondies d'assez grande taille de l'ordre du micromètre, à couches phospholipidiques superposées), myéline tubulaire (formation à symétrie quasiment cubique, de maille unitaire $\approx 50$ $\mathrm{nm}$, stabilisée par la présence de SPA), structures myéliniques (grandes structures présentant des convolutions en relation morphologique avec lc film superficicl) et vésicules uni- ou paucilamellaires de petite taille (quelques dizaines de nanomètres). Physiologiquement, les corps lamellaires se transformeraient en myéline tubulaire puis en structures myéliniques. Les vésicules seraient les formes d'élimination des phospholipides superficiels. Ce cycle est sans doute $m / s n^{\circ} 1$, vol. 7, janvier 91 généisation de la ventilation (par stabilisation des bronchioles et des alvéoles, diminution de la densité du tissu pulmonaire). L'hématose bénéficie de ces effets (augmentation de la surface alvéolaire, diminution de l'épaisseur de diffusion des gaz, renouvellement accru de l'air alvéolaire et homogénéisation du rapport ventilation-perfusion).

Il est connu depuis longtemps que la tension de surface est très basse dans les poumons, mais ce n'est qu'assez récemment que l'évolution de cette tension a pu être mesurée in situ $[8$, 9] (figure 2, p. 40). Deux particularités sont à souligner. D'une part, la tension de surface maximale $\gamma_{\text {mix }}$ est toujours modérée, de l'ordre de 28 $\mathrm{mN} / \mathrm{m}$ en fin d'inspiration en conditions statiques [8], inférieure ou égale à $40 \mathrm{mN} / \mathrm{m}$ [9] en conditions dynamiques (la tension du soluté salé isotonique à $37{ }^{\circ} \mathrm{C}$ est d'environ $71 \mathrm{mN} / \mathrm{m}$ ). D'autre part, en expiration, la surface alvéolaire diminuant, la tension interfaciale entre l'air et le liquide endoalvéolaire devient quasiment évanescente $\left(\gamma_{\text {min }}\right.$ proche de $0 \mathrm{mN} / \mathrm{m}$ ). Ces deux particularités du surfactant sont d'une importance capitale dans le comportement physiologique pulmonairc.

\section{Mécanismes tensio-actifs du surfactant (figure 3, p. 41)}

Le phénomène de tensio-activité est lié à la présence de molécules amphiphiles à l'interface, le plus souvent sous la forme d'une couche monomoléculaire. Deux groupes de mécanismes sont à différencier : les phénomènes intervenant dans la couche tensio-active et les échanges entre le milieu aqueux et la couche tensioactive elle-même.

Le surfactant présente la propriété de diminuer considérablement, lorsque le film est comprimé, la tension interfaciale existant entre l'air et l'eau. Ce comportement est lié à la présence de la DPPC - qui est en "phase gel bidimensionnel" à $37^{\circ} \mathrm{C}$. Cette couche superficielle, véritable phase solide créée à l'interface par la compression, supporte l'essenticl des contraintes mécaniques. Lorsque l'aire est fortement diminuée à l'interface, il se forme des objets tridimensionnels appelés struc- 


\section{RÉFÉRENCES}

11. Denizot BA, Tchoreloff P, LaunoisSurpas MA, et al. Exogenous pulmonary surfactant : influence of several physical treatments applied to liposomal suspension on experimental in vivo efficiency. 5th Cong Int Technol Pharm 1989 ; III : 48-53.

12. Denizot B. Surfactants pulmonaires exogènes : essai d'interprétation physicochimique de l'action pharmacologique. Thèse de doctorat d'université n⿳ 131 , faculté de pharmacie, université de ParisSud, 1990.

13. Chu J, Clements JA, Cotton EK, et al. Neonatal pulmonary ischemia. Part 1: clinical and physiological studies. Pediatrics $1967 ; 40$ : 709-82.

14. Obladen M, Schwartz H, Kattner E, Stevens P. Results with artificial surfactant : aspects of morphological appearance, surface activity, and in vivo activity of artificial surfactant. In : Herzog H, ed. Basic Research on Lung Surfactant, Progress in Respiration Research. Basel : Karger, 1990 : 247-52.

15. Yu SH, Harding GR, Possmayer F. Artificial pulmonary surfactant. Potential role for hexagonal HII phase in the formation of a surface-active monolayer. Biochim Biophys Acla 1984; 776 : 37-47.

16. Holm BA, Notter RH, Enhörning G. Biophysical and physiological properties of synthetic surfactants. In : Herzog $\mathrm{H}$, ed. Basic Research on Lung Surfactant, Progress in Respiration Research. Basel: Karger, 1990 : 305-7.

17. MacIver D, Possmayer F, Schürch S. A synthetic emulsion reproduces the functional properties of pulmonary surfactant Biochim Biophys Acla 1983 ; 751 : 74-80.

18. Holm BA, Enhörning $G$, Notter RH. A biophysical mechanism by wich plasma proteins inhibit lung surfactant activity. Chem Phys Lipids 1988 ; 49 : 49-55.

19. Morgenroth K, Batzenschlager A. Le système surfactant du poumon. Berlin : Walter de Gruyter and Co, 1988.

20. Dehan M, Denizot B. Les surfactants

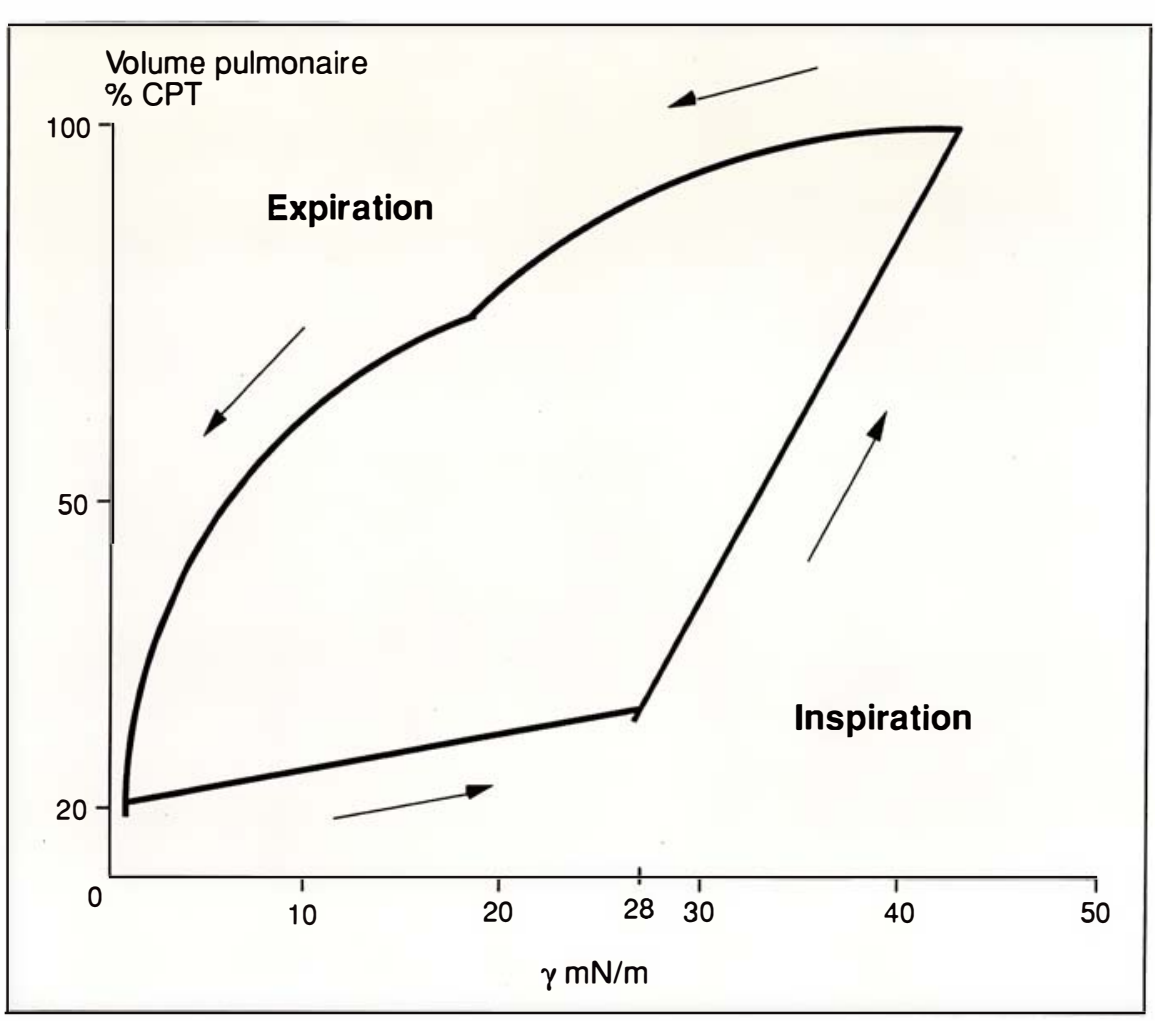

Figure 2. Évolution physiologique de la tension interfaciale in situ, $\gamma$, en fonction du volume pulmonaire. En inspiration (courbe inférieure), la tension interfaciale croît rapidement jusqu'aux environs de $28 \mathrm{mN} / \mathrm{m}$, pour n'augmenter que lentement au-delà. En fin d'expiration (courbe supérieure), à faible volume pulmonaire (exprimé en \% de la capacité pulmonaire totale CPT), une très basse tension $(<10 \mathrm{mN} / \mathrm{m})$ apparaît. Les flèches indiquent le sens de variation de $\gamma$ en fonction du volume. [D'après Smith et Stamenovic, J Appl Physiol $1986 ; 60$ : 1341-50.J

ture d'effondrement (collapse structures en anglais). Celles du surfactant naturel - que notre équipe a étudiées par une technique de prélèvement sur support solide et observation au microscope électronique (en collaboration avec Mme Gulick, Cnrs, Gif-sur-Yvette) ou diffraction d'un rayonnement $\mathrm{X}$ (en collaboration avec M. Benattar, CEA, Saclay) - présentent des caractéristiques de morphologie, de taille et d'organisation intermoléculaire très particulières qui les distinguent de phospholipides modèles* [10] telle la DPPC étudiée dans les mêmes conditions.

* Tchoreloff P, Gulik A, Denizot B, Proust JE, Puisieux F. Electron microscope study of superficial structures, obtained by compression of synthetic phospholipids monolayers, in comparison with natural lung surfactant. Poster at the " 60 years of surfactant research" congress, Rhine river, 11th-17th November 1989.
Ces structures ressemblent ainsi, par certains côtés, aux structures myéliniques présentes in vivo dans les alvéoles. Lors de l'accroissement de l'aire superficielle, ces structures obtenues à partir de surfactant naturel se réétalent facilement et libèrent leurs molécules tensioactives, à la différence de certains phospholipides modèles. Ce phénomène de réétalcment dépend des phases phospholipidiques en présence (phase lamellaire, cristal liquide ou gel), de l'hydratation des têtes polaires, des séparations de phase intervenant dans les mélanges. Les molécules ne se réétalant pas peuvent rester en surface, ou être perdues en formant des objets solubles dans la sous-phase, sous la forme de liposomes.

Le second mécanisme qui permet une activité superficielle efficace est 
contrôlé par les interrelations entre la sous-phase et la couche tensio-active elle-même: la perte de matériaux superficiels, inévitable lors des cycles de compression-expansion successifs, doit être en permanence compensée par l'arrivée de nouvelles molécules en surface (phénomène d'adsorption). Cette cinétique d'adsorption, déterminante pour l'efficacité des surfactants d'origine exogène chez l'animal [11], peut être accélérée par des phénomènes analogues à ceux favorisant la coalescence de suspensions phospholipidiques*. Dans le cas du surfactant naturel, ce rôle est joué par les apoprotéines B et C. Le mécanisme précis de l'adsorption à partir d'une suspension de liposomes n'est cependant pas connu : un des buts de notre équipe est de comprendre ce phénomène [12].

\section{Applications aux surfactants artificiels}

Parmi les surfactants artificiels proposés dans la littérature, peuvent être distinguées plusieurs générations de produits.

Dans les premiers essais, de la DPPC pure fut utilisée, en raison des propriétés de cette substance en état de compression; les résultats cliniques furent mauvais [13], en particulier parce que ces préparations ne possèdent pas les propriétés nécessaires de réétalement et d'adsorption rapide. Dans la seconde génération de surfactants artificiels, le phosphatidylglycérol (PG), constituant quasi spécifique du surfactant naturel, fut ajouté. Là encore, les différents mélanges DPPC-PG testés, sous forme de poudre ou de liposomes [14], ne présentèrent pas de caractéristiques suffisantes de réétalement ni, surtout, d'adsorption rapide. La même remarque s'applique à un surfactant artificiel développé il y a quelques années par notre équipe, constitué d'un mélange DPPC-distéaroylphosphatidylcholine-lécithine de soja [11].

\footnotetext{
* Denizot B, Tchoreloff P, Proust J, Lindenbaum A, Dehan $M, 1989$. Thermodynamic parameters of $D O P C$ liposomes adsorption rate at an air-water interface. Poster in the "60 years of surfactant research" congress, Rhine river, 11th-17th November 1989. $\mathrm{m} / \mathrm{s} n^{\circ} 1$, vol. 7, janvier 91
}

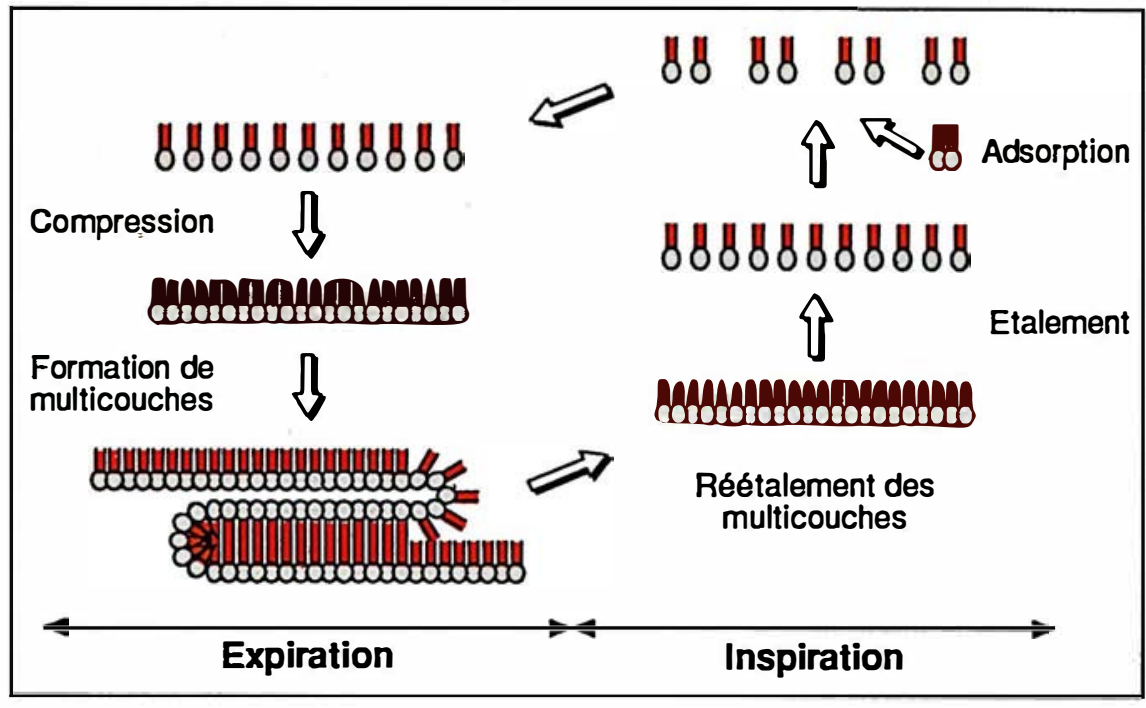

Figure 3. Schématisation des phénomènes tensio-actifs intervenant au cours du cycle ventilatoire. En expiration, les molécules superficielles de surfactant sont comprimées et forment des structures multilamellaires constituées de couches superposées. En inspiration, ces structures se réétalent, puis de nouvelles molécules sont recrutées à partir de la sous-phase (adsorption).

La troisième génération de surfactant artificiel à développement clinique est caractérisée par la présence de composants non phospholipidiques. La présence d'hexadécanol dans l'Exosurf $^{\circledast}$ - seul représentant actuel permet, en perturbant la structure bilamellaire de la DPPC, d'accroître notablement les performances d'adsorption mais surtout de réétalement.

De nombreux surfactants artificiels n'ont pas eu de développement clinique malgré de bonnes performances in vitro. Ce fut le cas des mélanges DPPC-phosphatidyl éthanolamine [15], DPPC-acides gras et/ou triglycérides [16] ou de la DPPC émulsifiée par des huiles naturelles ou artificielles [17], qui, in vitro, forment aisément des phases non lamellaires. L'inefficacité, in vivo, de ces préparations est sans doute liée à leur inhibition par les protéines plasmatiques [18] qui s'adsorbent de façon compétitive à la surface alvéolaire. Dans notre laboratoire sont en développement de nouvelles formulations, fondées sur la compréhension des mécanismes tensio-actifs et optimalisant les performances de réétalement et d'adsorption.
Pour obtenir des surfactants artificiels plus opérationnels, il faudra aussi prendre en compte les propriétés non tensio-actives du surfactant au niveau tant alvéolaire que bronchiolaire. On sait, par exemple, que la présence d'un film superficiel permet de ralentir l'évaporation locale, protégeant ainsi la muqueuse contre le dessèchement excessif, d'établir une clairance mucociliaire [19] en induisant un plan de clivage entre une phase mucus-sol et une phase mucus-gel (figure 4, p. 42), et de jouer sans doute un rôle d'anti-adhésif. Le surfactant présente également des fonctions biochimiques, phagocytaires et immunologiques.

\section{Conclusion}

L'étude approfondie des propriétés du surfactant pulmonaire est un domaine de recherche récent. Les résultats ont permis de proposer, dans le cadre des détresses respiratoires dues à une déficience en surfactant telle la maladie des membranes hyalines, un traitement ayant une certaine efficacité [20]. Toutefois, de nouvelles préparations plus performantes doivent être proposées à par- 


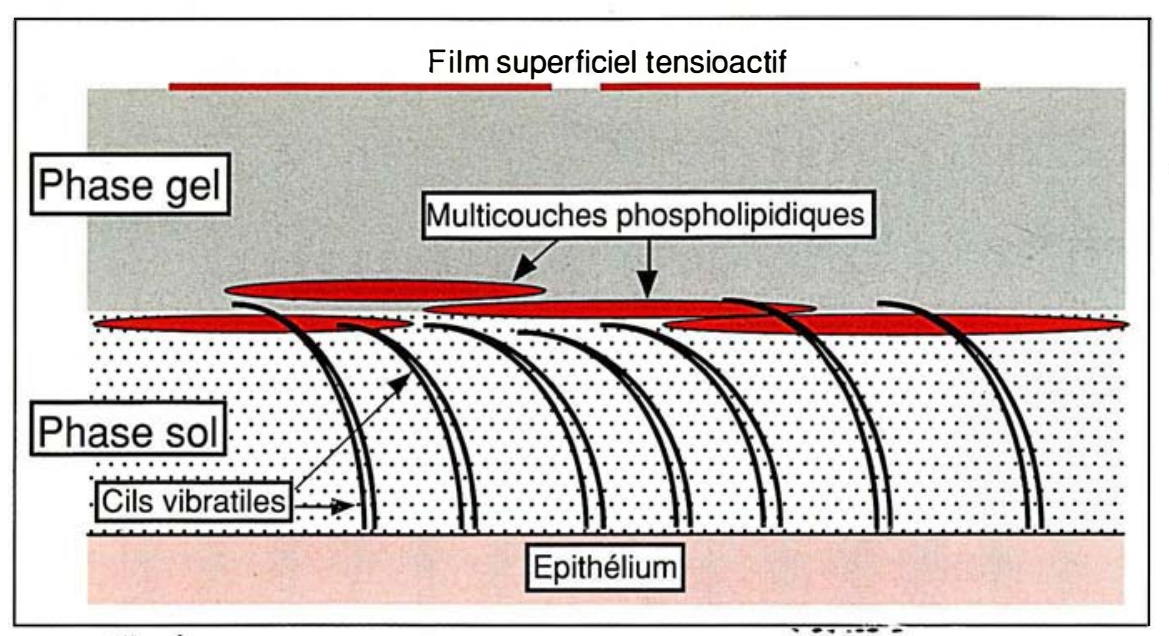

Figure 4. Morphologie de la surface bronchiolaire. Entre la surface épithéliale et le milieu gazeux se trouvent deux couches de mucus, séparées par des multicouches phospholipidiques : une phase superficielle, solide, de structure gélifiée, et une phase sol, liquide, dans laquelle battent les cils vibratiles. La présence de surfactant autorise la formation d'un plan de clivage et la mobilisation de la phase gel. (Schéma réalisé d'après [19].)

tir des connaissances fondamentales des mécanismes régissant la tensioactivité mais aussi des autres propriétés du surfactant naturel.

Les recherches entreprises dépassent le cadre de la maladie des membranes hyalines et concernent d'autres maladies respiratoires tels l'état de mal asthmatique ou les syndromes de détresse respiratoire de l'adulte. Les surfactants exogènes jouent ici un rôle en traitant le malade mais aussi en aidant à discerner la part due au système surfactant et celle liée aux autres composantes physiopathologiques (lésions vasculaires, chimiques...).

Enfin, l'utilisation de la voie intrapulmonaire pour l'administration de médicaments à visée systémique est un objectif à plus long terme et les recherches sont encore, en ce domaine, très préliminaires : la formulation de nouveaux surfactants y est un préalable indispensable à des avancées importantes

\section{Remerciements}

Les auteurs tiennent à remercier l'Inserm (contrat de recherche externe $\mathrm{n}^{\circ} 894011$ ) et la Fondation de France pour leur support financier.

\section{Summary}

Tensio-active mechanisms at surface of pulmonary alveoli

The hyaline membrane disease of the premature newborn is due to a deficiency of the pulmonary surfactant related to lung biochemical immaturity. The exogenous surfactant therapy tries to compensate the lack of tension-activity at the air-endoalveolar fluid interface.

This article reviews the present knowledge on biophysical parameters of the surfactant, the interaction of which governs the physiological pulmonary behavior. The new notions about the mechanisms intervening in the tensio-active actions have important consequences on the formulation of the recently developed artificial therapeutic preparations. 